\title{
Development and tests of fast 1-MA linear transformer driver stages
}

\author{
A. A. Kim, ${ }^{1}$ M. G. Mazarakis, ${ }^{2}$ V. A. Sinebryukhov, ${ }^{1}$ B. M. Kovalchuk, ${ }^{1}$ V. A. Visir, ${ }^{1}$ S. N. Volkov, ${ }^{1}$ F. Bayol, ${ }^{3}$ \\ A. N. Bastrikov, ${ }^{1}$ V. G. Durakov, ${ }^{1}$ S. V. Frolov, ${ }^{1}$ V. M. Alexeenko, ${ }^{1}$ D. H. McDaniel, ${ }^{2}$ W. E. Fowler, ${ }^{2}$ K. LeChien, ${ }^{2}$ \\ C. Olson, ${ }^{2}$ W. A. Stygar, ${ }^{2}$ K. W. Struve, ${ }^{2}$ J. Porter, ${ }^{2}$ and R. M. Gilgenbach ${ }^{4}$ \\ ${ }^{1}$ Institute of High Current Electronics, Russian Academy of Sciences, Tomsk 634055, Russia \\ ${ }^{2}$ Sandia National Laboratories, Albuquerque, New Mexico 87185, USA \\ ${ }^{3}$ International Technologies for High Pulsed Power, Thégra 46500, France \\ ${ }^{4}$ University of Michigan, Ann Arbor, Michigan 48109-2104, USA
}

(Received 4 March 2009; published 29 May 2009)

\begin{abstract}
In this article we present the design and test results of the most powerful, fast linear transformer driver (LTD) stage developed to date. This 1-MA LTD stage consists of 40 parallel RLC (resistor R, inductor L, and capacitor C) circuits called "bricks" that are triggered simultaneously; it is able to deliver $\sim 1$ MA current pulse with a rise time of $\sim 100 \mathrm{~ns}$ into the $\sim 0$. 1-Ohm matched load. The electrical behavior of the stage can be predicted by using a simple RLC circuit, thus simplifying the designing of various LTD-based accelerators. Five 1-MA LTD stages assembled in series into a module have been successfully tested with both resistive and vacuum electron-beam diode loads.
\end{abstract}

DOI: 10.1103/PhysRevSTAB.12.050402

PACS numbers: $29.20 . \mathrm{D}-$, 84.30.Ng

\section{INTRODUCTION}

The linear transformer driver (LTD) is a developing pulsed-power technology which shows promise for applications that require high-power, high-voltage, highcurrent, $\sim 100$-ns output pulses. The LTD driver is an induction generator similar to the linear induction accelerator (LIA) [1,2], the inductive voltage adder (IVA) [3-6], and the linear pulsed transformer (LPT) [7]. All induction generators are based on Faraday's law which states that the time-varying magnetic flux $B(t)$ penetrating a loop generates at the ends of this loop a voltage difference $U(t)$ equal to

$$
U(t)=S \frac{d B}{d t},
$$

where $S$ is the cross section of the loop. In an induction generator normally the loop is realized as a pillbox-shaped toroid, which in this article is called "induction cavity." This toroid has a continuous azimuthal gap, and the voltage $U(t)$ appears across this gap. Since historically the first devices utilizing Faraday's law were called "transformers," all induction generators can also be considered as transformers. Hence, the induction cavity can be considered as the single-turn secondary winding of such a transformer. Its primary winding, which generates the azimuthal magnetic flux $B(t)$ that penetrates the cavity, may have different designs. A LIA is composed of a series of identical induction cavities. When assembled, it has a cylindrical region on axis, referred to as the bore, which has a series of annular gaps regularly spaced on its inner cylindrical surface. This bore is threaded by a beam of propagating particles which gets accelerated as they drift through each of the cavity gaps. The voltage across a given gap appears in coincidence with the passage of the particles through the gap. Hence, the firing sequence of each LIA cavity is synchronized with the speed the particles propagate along the axis of the device.

IVA is also formed by a series of identical induction cavities, but unlike in LIA, its bore is threaded by a center conductor that together with the cylindrical surface of the bore form an output line that connects to the load. The outstanding advantage of the IVA is that this line serves as a transmission line; i.e., the electromagnetic (EM) energy propagates along this line from each cavity towards the load only, without reflections. In an ideal IVA two fundamental principles must be satisfied: first, the firing of the successive cavities must be synchronized with the speed in which the EM wave propagates along the output line, and second, the wave impedance of the output line must gradually increase to be matched with the drive impedance of the upstream cavities. In an ideal IVA [excluding IVAs driving magnetic insulated transmission lines (MITLs)], the output voltage pulse across a matched load is independent of the number of cavities and is equal to the sum of all cavity pulses.

LPT can be formed by one or a series of identical induction cavities. If it is one cavity only, it encloses several parallel primary windings that are triggered simultaneously. The gap of this cavity is located not on the inner surface of the toroid but on its side surface, and it must be insulated for the total output voltage. Since all primary windings are triggered simultaneously, the discharge circuit of the single-cavity LPT consists of the primary energy sources that are connected in series to the load through the inductance of the toroid. This inductance widens the output pulse, and therefore the output pulse of the single-cavity LPT is not the sum of the primary pulses applied to each primary winding. An IVA-type device consisting of several 
induction cavities, where one or both of the above listed fundamental IVA principles are violated, is also an LPT in a sense that its output pulse is not a sum of the primary pulses. If any IVA principles are violated, the output line does not serve as a transmission line because the waves undergo multiple reflections; then the shape of the output pulse depends on the number of the cavities and on the width of the primary pulses. In particular, if the length of the primary pulses is much longer than the electrical length of the output line, this line serves as a series inductance, exactly in the same way as the inductance of the toroid in the single-cavity LPT.

Similar to IVA and LPT, the LTD driver consists of several induction cavities called "LTD stages" plus the center electrode threading through the axis of the cavities. The main difference from LIA, IVA, and LPT induction cavities is that the LTD stage encloses the primary capacitive energy storage [8]. The LTDs have several important advantages that make this technology attractive for the next generation of pulsed-power systems: (i) Since the LTD stages enclose the primary storage, the LTD driver is more compact compared to other generators having similar output parameters. For example, the footprint of a 1-TW, 10 LTD cavity driver is only $\sim 8 \mathrm{~m}^{2}$. (ii) As any other primary energy storage, the LTD driver is simple. As described below, an LTD stage consists mainly of only three different parts: a capacitor, a gas spark switch, and a ferromagnetic core. (iii) Since the LTD primary energy storage is enclosed inside the cavity, it is practical and convenient to be built with relatively small size capacitors, which necessarily have less capacitance $C$. But this turns to be an important advantage for LTDs since the small (10$40 \mathrm{nF}$ ) capacitance becomes instrumental for directly generating short output pulses even if the inductance of each of the basic circuit ("brick") may be relatively large $(\sim 250 \mathrm{nH})$. Each "brick," described in detail in Sec. II, consists of two capacitors and one switch connected in series. A high-current LTD cavity encloses many such bricks connected in parallel to the load. (iv) Since the LTD driver consists of several individually triggerable stages, it can be operated in both LPT and IVA modes.

All the above advantages of the LTD technology offer the capability [9-15] to build fast, 100-ns time-scale compact and simple high-power drivers without any intermediate power amplification stages.

In Sec. II of this paper we describe the design of the most powerful, fast, 1-MA LTD stage developed to date. In addition, the LTD cavity electrical performance and experimental results are discussed and compared with numerical circuit code calculations. Some peculiarities related to the behavior of the ferromagnetic core, depending on the premagnetizing methods, are also presented and explained in detail. In Sec. III we present the design and test results of the five-stage 1-MA LTD module driving both resistive and electron-beam diode loads in the LPT mode.

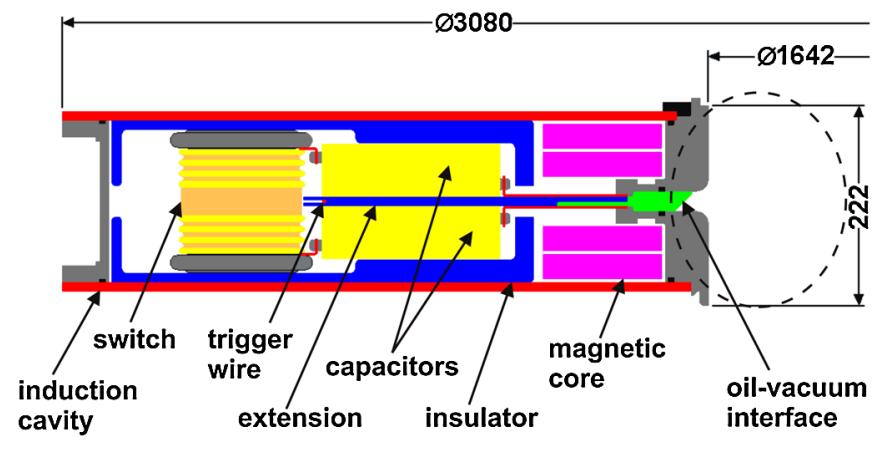

FIG. 1. (Color) Design of the 1-MA LTD stage showing two storage capacitors, the switch and the buses of the brick. Broken ellipse indicates the region of the load location and is presented in more detail in Fig. 2.

\section{FAST 1-MA LTD STAGE}

The design of the 1-MA fast LTD stage is shown in Fig. 1. The stage induction cavity is a pillbox-shaped toroid with an azimuthal gap at the center of the inside cylindrical surface. This gap is fitted with a vacuum tight oil-vacuum interface. Inside the cavity is located the capacitor bank with the switches, charge and trigger resistors, and the ferromagnetic core. The outside diameter of the stage is $\sim 3 \mathrm{~m}$, the length along the main axis $\sim 22 \mathrm{~cm}$, and the weight filled with oil $\sim 2.5$ tons.

The capacitor bank is made of 80 storage capacitors type GA $35426(40 \mathrm{nF}, 100 \mathrm{kV})$ [16] that are arranged in 40 pairs. The capacitors in each pair are charged in opposite polarity up to $\pm 100 \mathrm{kV}$ and switched into the load by closing the multispark gap switch type fast LTD [17]. The set of two capacitors, the switch, and the buses connecting the capacitors from one side with the switch and from the opposite side with the azimuthal gap is called brick and represents the basic element of the stage. All the bricks in the stage are connected in parallel and located evenly in a circular array around the axis. For safety reasons, the neighboring bricks are separated by $\sim 1 \mathrm{kOhm}$ charging resistors both in the positive and negative charging circuits.

The triggerable switches type fast LTD operate with dry air at a pressure of $\sim 4$ ata (or $0.405 \mathrm{MPa}$ ) for $\pm 100 \mathrm{kV}$ charging voltage. The DC voltage is distributed between several switch gaps with a corona discharge. Some features of these switches are described elsewhere [18-20]. They are triggered by $\sim-100 \mathrm{kV}, \sim 30 \mathrm{~ns}$ rise time pulses which enter into the stage via four $65-\mathrm{Ohm}$ high-voltage cables. Inside the stage, these cables are connected to a 4$\mathrm{mm}$ diameter circular trigger wire located in a groove at the outer diameter of the insulator extension of the oil-vacuum interface. The trigger electrodes of the switches are connected to this wire through $\sim 500 \mathrm{Ohm}$ resistors.

In the tests of the single stage described below, the $\sim 2$-m outer diameter ferromagnetic core had four separate rings, each ring being wound with a $80-\mu$-thick, $18-\mathrm{mm}$ - 
wide ET3524 anisotropic iron tape sandwiched in a 12- $\mu$-thick, 22-mm-wide Mylar film insulation. The rings are molded into an epoxy compound to stabilize the turns and isolate the rings from each other. The magnetic induction at saturation of ET3425 iron is $B_{S}=2 \mathrm{~T}$ at $H=$ $30 \mathrm{kA} / \mathrm{m}$, the residual induction is $B_{R}=1.2 \mathrm{~T}$, the coercive force is $H_{C}=0.03 \mathrm{kA} / \mathrm{m}$, and the specific resistance of the ET3425 iron is $\rho=5 \times 10^{-7} \mathrm{Ohm} \mathrm{m}$. The total cross section of the iron in each ring is $\sim 14 \mathrm{~cm}^{2}$. The volt-second integral of the entire core is $V S_{C}^{A} \sim 22.4 \mathrm{mVs}$ with active premagnetizing $(\Delta B=4 \mathrm{~T})$ and $V S_{C}^{P} \sim$ $18 \mathrm{mVs}$ with passive premagnetizing $(\Delta B=3.2 \mathrm{~T})$, where $\Delta B$ is the total variation of the induced magnetic field in the cores. The performance of the core depending on the premagnetizing methods is discussed in Appendix A.

The high-voltage components inside the cavity are insulated from the walls of the cavity and from each other with dielectric polyethylene insulators (please see Fig. 1). The 1-cm-thick extension of the oil-vacuum interface isolates the capacitors and planar output lines (buses) of the bricks. The oil-vacuum interface is made from extruded acrylic. After assembly the induction cavity is filled with transformer oil for additional electrical isolation of the components.

For the experimental tests of each individual 1-MA LTD stage, the resistive load was placed inside the cylindrical opening of the stage as shown in Fig. 2. Two concentric plastic polyethylene cylinders were inserted separating the stage's inner cylindrical cavity into 2 sections. These sections were hermitically sealed up and down with two metallic conducting plates attached to the outer planar walls of the stage. 20 plastic rods were utilized to compress the "O" rings and establish electrical contact between the plates and the walls of the stage. The first of those two

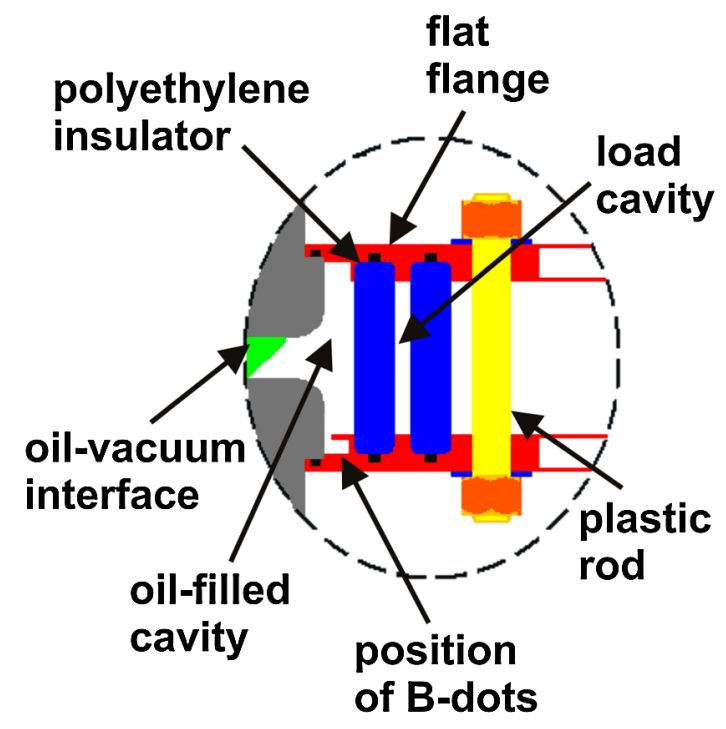

FIG. 2. (Color) Design of the load in tests of separate stage.

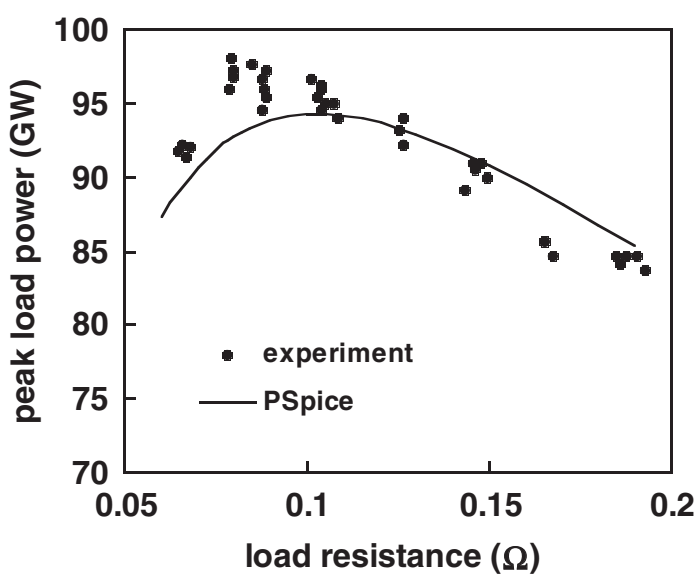

FIG. 3. Peak load power dependence on the load resistance. The dots are the experimental results and are compared with PSPICE simulation (solid curve).

sections, which is closest to the oil-vacuum interface, was filled with oil to avoid flashing along the interface surface. The second cavity between the two cylindrical insulators was filled with $\mathrm{NaCl}$-water solution and served as the resistive load of the stage. The inductance of such load, estimated from its geometry, was $1.05 \mathrm{nH}$.

In these tests, the load current, $I$, was measured with two magnetic flux monitors $(d B / d t)$ wound in opposite directions to produce opposite-polarity signals for electrical noise rejection and placed in the vicinity of the load. The load voltage $U$ was measured with an external voltage divider, and the current around the core $I_{C}$ was measured with another magnetic flux monitor $(d B / d t)$. The resistance of the load defined as $R=U / I$ was varied from $\sim 0.06$ to $\sim 0.19 \mathrm{Ohm}$ by changing the concentration of the $\mathrm{NaCl}$ solution. The core was premagnetized before the shot (passive premagnetizing, see Appendix A) by using the premagnetizing pulse generator (PPG). The PPG is essentially an LC circuit with an inductance $L=20 \mu \mathrm{H}$ and capacitance $C=40 \mu \mathrm{F}$. It was charged to $2 \mathrm{kV}$. A diode was connected in parallel to the capacitor in order to eliminate oscillations of the PPG voltage across the core.

Experiments were conducted with different resistive loads at $\pm 100 \mathrm{kV}$ charging of the LTD capacitors. The obtained experimental results are summarized in Figs. 3-6 and are compared with PSPICE ${ }^{1}$ [21] simulations. Figure 3 indicates that the optimum output stage impedance [15], which maximizes the output power to $\sim 96 \mathrm{GW}$, is $R \sim$ $0.1 \mathrm{Ohm}$. In this case, the load voltage (Fig. 4) is $\sim 100 \mathrm{kV}$ and the power rise time (Fig. 5) is $\sim 90$ ns. The simulation is in a reasonable agreement with experimental results; the only noticeable difference is the faster decrease of the energy delivered to the load for $R>0.1 \mathrm{Ohm}$ as compared with the simulation prediction (Fig. 6). The energy is calculated according to equation

\footnotetext{
${ }^{1}$ PSPICE is a registered trademark of MicroSim Corporation.
} 


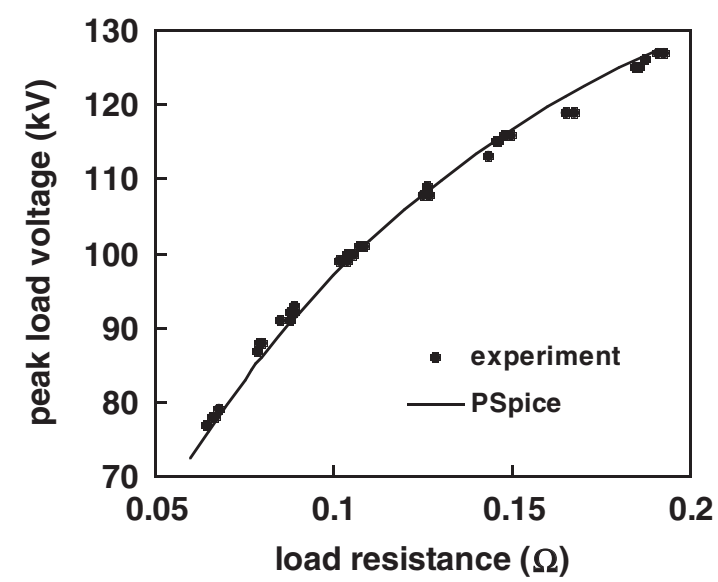

FIG. 4. Peak load voltage dependence on the load resistance. The dots are the experimental results while the solid curve is the PSPICE simulation.

$$
E=\int_{0}^{t_{0}} U I d t
$$

where $t_{0}$ is the time when the load voltage crosses zero for the first time. The experimentally observed energy $E$ has a maximum of $\sim 11.3 \mathrm{~kJ}$ occurring for a load resistance $R \sim$ $0.1 \mathrm{Ohm}$ and is equal to $\sim 70 \%$ of the total stored energy in the capacitors. In PSPICE, the energy $E$ also peaks close to $R \sim 0.1 \mathrm{Ohm}$, but for larger load resistances does not decrease as fast as in the experiments. The cause of this difference will be discussed below.

Figure 7 shows the recorded load voltage $U$ and the current $I_{C}$ leaking around the core to the cavity walls for $R=0.107 \mathrm{Ohm}$. At such load the volt-second integral $V S_{\mathrm{LTD}}$, calculated as

$$
V S_{\mathrm{LTD}} \sim \int_{0}^{t} U d t
$$

does not reach the limit $V S_{C}^{P}=18 \mathrm{mVs}$ (please see Fig. 13

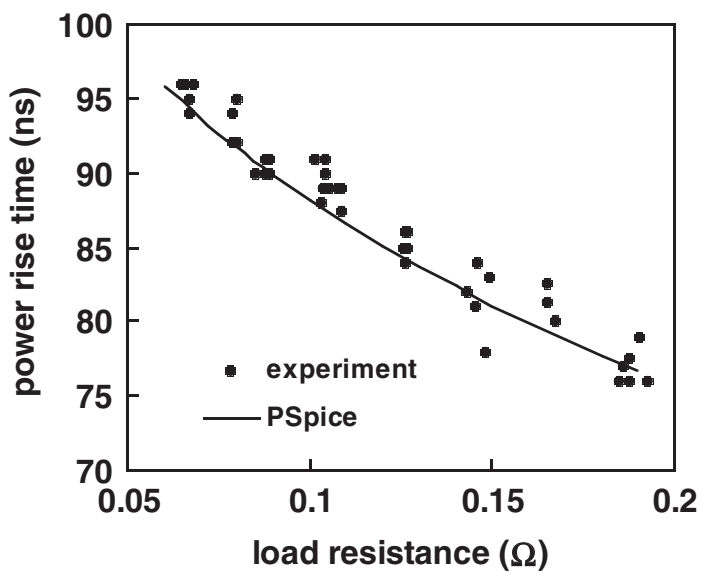

FIG. 5. Power rise time dependence on the load resistance. The experimental data points are compared with the PSPICE numerical simulation (solid curve).

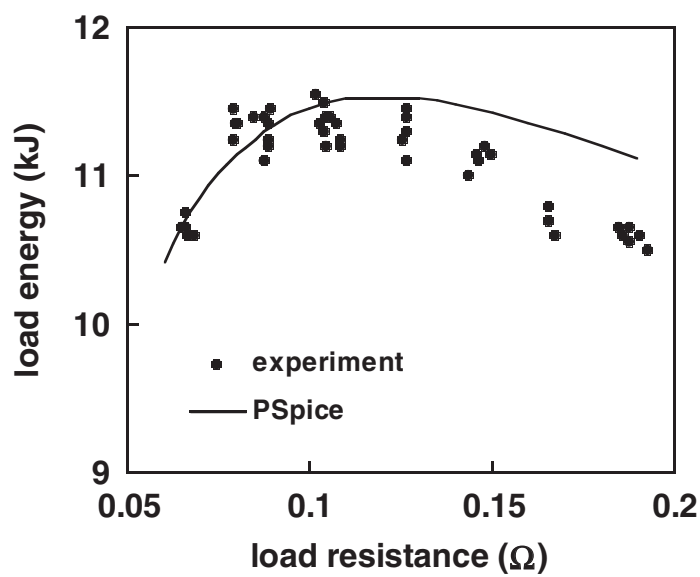

FIG. 6. Energy delivered to the load during the main pulse as a function of the load resistance. The experimental results (dots) are compared with the PSPICE simulation (solid curve).

of Appendix A). The shape of the current $I_{C}$ is approximately the same as that of the voltage, indicating that the core in this shot behaved as a constant resistance $R_{C}=$ $U / I_{C}$. For a measured load voltage $U \sim 100 \mathrm{kV}$ and $I_{C} \sim$ $180 \mathrm{kA}$, the value of this resistance is estimated to be $R_{C} \sim$ $0.56 \mathrm{Ohm}$.

Figure 8 presents similar traces as in Fig. 7, but now the results are obtained with an $R \sim 0.188 \mathrm{Ohm}$. In this case the observed load voltage is higher and $V S_{\text {LTD }}$ exceeds $V S_{C}^{P}=18 \mathrm{mVs}$ after $\sim 200 \mathrm{~ns}$. At that time, the current $I_{C}$ begins to rise again, confirming the saturation of the core. In spite of the higher load resistance, the voltage reversal here is larger than that of Fig. 7 because the inductance of the loop around the core, connected in parallel to the load, becomes very small and acts practically as a short.

The performance of the core depending on the premagnetizing methods was studied at $R=0.166 \mathrm{Ohm}$ (Fig. 9). With this load and passive premagnetizing the core satu-

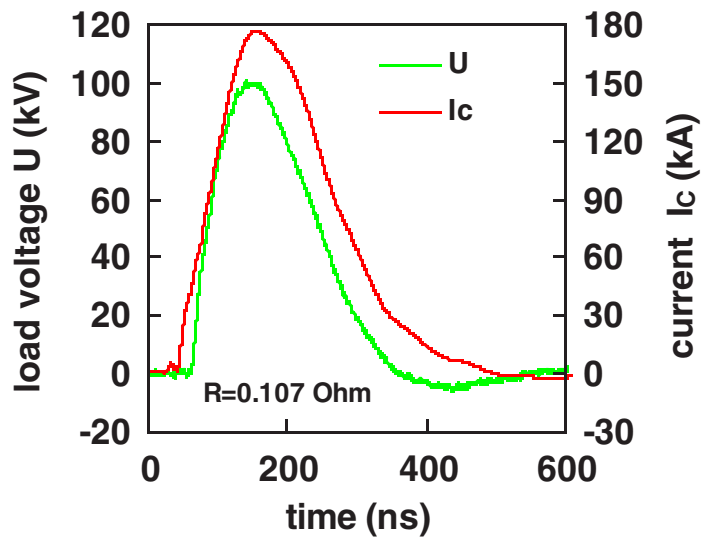

FIG. 7. (Color) Load voltage $U$ and current around the core $I_{C}$ at $R=0.107 \mathrm{Ohm}$. The volt-second integral $V S_{\mathrm{LTD}}$ does not reach the maximum of $V S_{C}^{P}=18 \mathrm{mVs}$. The current $I_{C}$ is approximately proportional to the voltage $U$. 


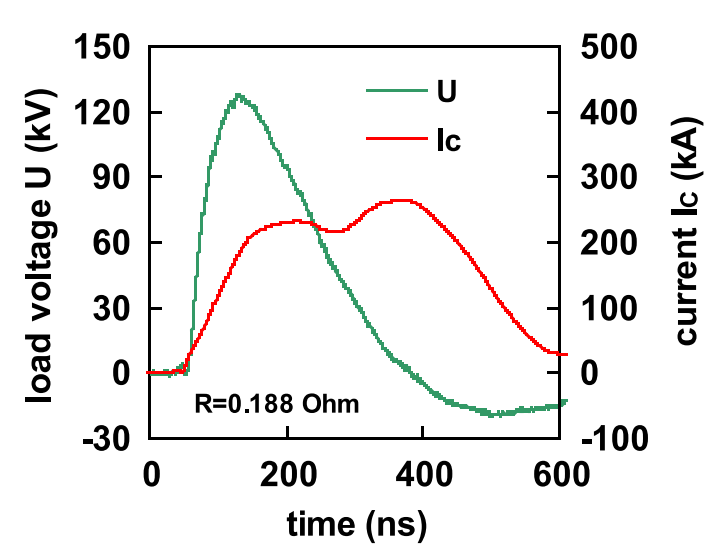

FIG. 8. (Color) Same traces as in Fig. 7 but with a different $R=$ $0.188 \mathrm{Ohm}$. Because of higher voltage $U$ the volt-second integral $V S_{U}$ exceeds the limit $V S_{C}^{P}=18 \mathrm{mVs}$ at $\sim 200 \mathrm{~ns}$ in the pulse. At that time the current $I_{C}$ begins to rise again, indicating saturation of the core.

rates at $\sim 200 \mathrm{~ns}$ when $V S_{\mathrm{LTD}}$ reaches $V S_{C}^{P}=18 \mathrm{mVs}$. The energy loss in the core is $\sim 3.6 \mathrm{~kJ}$. At active premagnetizing the volt-second integral $V S_{\mathrm{LTD}}$ reaches again the value of $18 \mathrm{mVs}$ at the same time, but now it does not reach the maximum available value of $V S_{C}^{A}=22.4 \mathrm{mVs}$. In this case, since the core does not saturate the energy losses are smaller and equal to $3.3 \mathrm{~kJ}$, a decrease of $300 \mathrm{~J}$. When the core is saturated from the very beginning, the current around the core is similar to the current through an inductor (e.g. it peaks at approximately the same time as the voltage crosses zero). The current exceeds $800 \mathrm{kA}$, the load voltage amplitude decreases by $\sim 10 \%$, the total length of the pulse

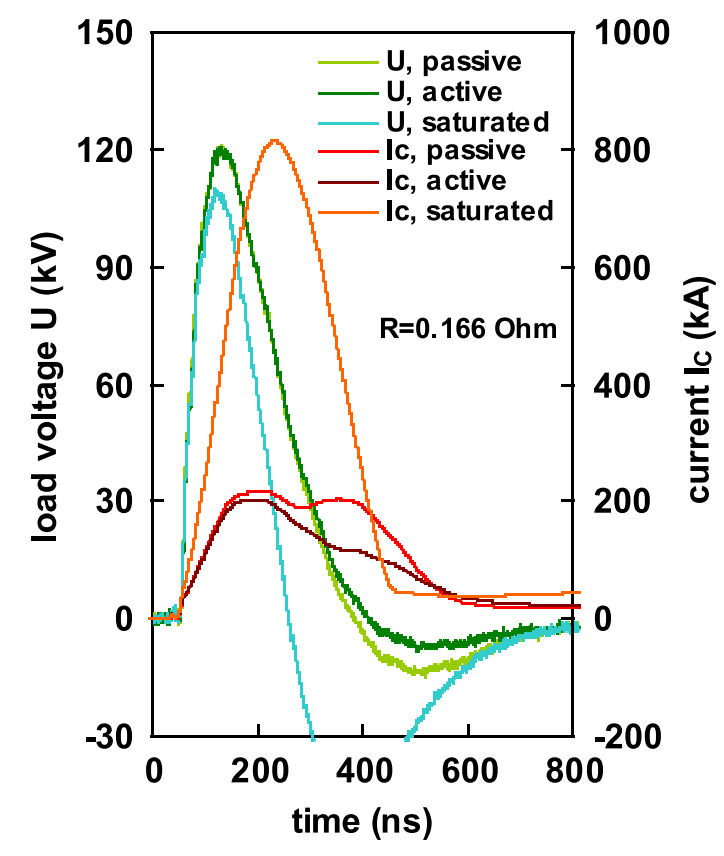

FIG. 9. (Color) Load voltage $U$ and current around the core $I_{C}$ at $R=0.166$ Ohm depending on the premagnetizing method.

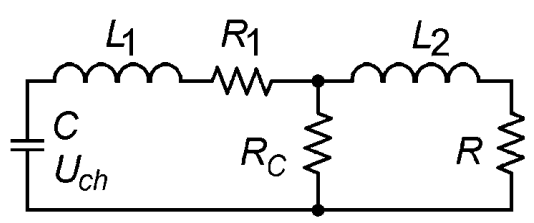

FIG. 10. Equivalent electrical circuit of a fast LTD stage.

decreases from $\sim 350 \mathrm{~ns}$ to $\sim 200 \mathrm{~ns}$, and the energy loss by the time the voltage crosses zero increases to almost $\sim 6 \mathrm{~kJ}$.

Assuming that all switches close simultaneously and the cores do not saturate, the discharge of an LTD stage can be represented by a simple equivalent RLC (resistor R, inductor L, and capacitor C) circuit (Fig. 10). Here $C$ is the total storage capacitance of all the bricks connected in parallel; $R_{1}$ is the resistance of the capacitors and the switches, $L_{1}$ is the total inductance of the bricks, and $L_{2}$ and $R$ are the inductance and the resistance of the load.

The resistance $R_{C}$ simulates the LTD core before it saturates. For slow pulses, the core behaves as a variable inductor $L_{\mu}$, which is proportional to magnetic permeability of the core material $\mu=d B / d H$. In this case the circuit in Fig. 10 should include $L_{\mu}$ in parallel to the load instead of the $R_{C}$. The energy loss in the core would be defined by the surface area inside the static hysteresis loop $B=f(H)$. The volume of the 1-MA LTD core is $W \sim 0.036 \mathrm{~m}^{3}$, then for the parameters of the ET3425 iron, the energy loss in $L_{\mu}$ at maximum possible $\Delta B=2 B_{S}$ is $E \sim W\left(2 H_{C}\right) \times$ $\left(2 B_{S}\right)<20$ J. (Please see Fig. 13)

If the voltage pulse applied to the conductive core is fast enough, it generates eddy current in the core material. The value of this current is defined by the driving voltage and the resistance of the layer which carries eddy current, $R_{C}$. However, to be precise even for the fast pulses, the circuit of Fig. 10 should include $L_{\mu}$ in parallel with the resistance $R_{C}$. Generally speaking, the resistance $R_{C}$ is also variable since it reflects the dynamics of eddy current penetration into the core iron. Though the physics of this penetration is clearly the same as that of the skin depth, the exact expression of $R_{C}$ for the given shape of the voltage pulse is rather complicated. For engineering purposes, the resistance $R_{C}$ can be calculated assuming [as in Ref. [22]] that the static hysteresis curve and the voltage pulse are rectangular or [as in Ref. [23]] that $d B / d t$ is approximately constant everywhere throughout the tape. Both approaches result in a constant value given by

$$
R_{C}=k \frac{\rho S}{\delta^{2} \ell}
$$

where $\rho$ is the specific resistance of the core iron, $S$ the total cross section of the iron in the core, $\ell$ the length of the core, and $\delta$ the thickness of the iron tape. The dimensionless coefficient $k$ is found to be 8 in [22], and to be 12 in [23]. The dominant branch among $L_{\mu}$ and $R_{C}$ can be 
evaluated by comparing the total current $I_{C}(t)$ flowing around the core with the voltage across the load $U(t)$ (which is approximately the same as the voltage across $L_{\mu}$ and $R_{C}$ ). As discussed above, the traces in Fig. 9 indicate that if the core is not saturated the current $I_{C}(t)$ is almost proportional to the voltage $U(t)$, i.e., in the unsaturated core the resistance $R_{C}(\sim 0.56 \mathrm{Ohm})$ dominates. This value is rather close to $R_{C} \sim 0.7 \mathrm{Ohm}$ calculated from the Eq. (4) with $\delta=80 \mu, S=56 \mathrm{~cm}^{3}$, $\rho=5 \times 10^{-7} \mathrm{Ohm} \mathrm{m}, \ell=6 \mathrm{~m}$, and $k \sim 10$. For a sinusoidal voltage pulse with the peak amplitude of $\sim 100 \mathrm{kV}$, the energy loss during the first quarter period $T / 2$ $(\sim 350 \mathrm{~ns})$ in a core resistance $R_{C} \sim 0.6-0.7 \mathrm{Ohm}$ is $2.5-3 \mathrm{~kJ}$, which is much higher than the maximum possible energy loss in $L_{\mu}(<20 \mathrm{~J})$. Therefore the inductance $L_{\mu}$ in the circuit of Fig. 10 is neglected. (Note that when the core saturates, the value of $\mu$ drastically decreases and the circuit should include the greatly reduced $L_{\mu}$ rather than the $R_{C}$. (See the voltage and current traces for the saturated core case of Fig. 9). The best fits of the experimental data with the PSPICE code simulations are presented in Figs. 3-6. They were obtained using the circuit of Fig. 10 with $U_{\mathrm{CH}}=200 \mathrm{kV}, C=800 \mathrm{nF}, R_{1}=0.0165 \mathrm{Ohm}, R_{C}=$ $0.65 \mathrm{Ohm}, L_{1}=6 \mathrm{nH}$, and $L_{2}=1.05 \mathrm{nH}$. These values are the total equivalent capacitance, inductance, and resistance of all of the 40 bricks of the 1-MA LTD stage. Here the inductance and resistance of the brick is assumed to be $240 \mathrm{nH}$ and $0.66 \mathrm{Ohm}$, respectively. Since the simulated curves in Figs. 3-5 are quite close to the experimental data, we can conclude that the jitter of the 40 switches is at least much less than the rise time of the output pulse.

The difference between the simulated and observed energy delivered to the load for $R>0.1 \mathrm{Ohm}$ (Fig. 6) seems to be the result of saturation of the core, which is not taken into account by the simple RLC circuit of Fig. 10. For example, at $R=0.166 \mathrm{Ohm}$ (Fig. 9) the saturation of the core at passive premagnetizing increases the energy loss in the core by $\sim 0.3 \mathrm{~kJ}$. If this increase is subtracted from the simulated energy $E=11.3 \mathrm{~kJ}$ of Fig. 6, the energy delivered to the load becomes $E \sim 11 \mathrm{~kJ}$, which is much closer to what was measured in the experiment.

Note that, for the circuit in Fig. 10, the optimum load impedance providing the maximum output power can be calculated from the expression:

$$
R_{\mathrm{opt}}=R^{*}\left[1-0.73\left(1-0.66 \frac{L_{2}}{L_{1}}\right) \frac{R^{*}}{R_{C}}\right],
$$

where

$$
R^{*}=1.1 \sqrt{\frac{L_{1}+L_{2}}{C}}+0.8 R_{1}
$$

is the optimum load impedance for the case $R_{C} \rightarrow \infty$ [15]. Equation (5) is correct to within $\sim 1 \%$ whenever
$\frac{R_{1}}{\sqrt{\left(L_{1}+L_{2}\right) / C}}<0.5, \frac{R_{\mathrm{opt}}}{R_{C}} \leq 0.2$, and $\frac{L_{2}}{L_{1}} \leq 0.3$. A derivation of this expression is given in Appendix B.

The experimental results presented above were obtained with the first prototype 1-MA LTD stage. The loss current around the core when the stage operates at almost peak power is $\sim 180 \mathrm{kA}$, which is $\sim 19 \%$ of the load current $I=$ $U / R \sim 935 \mathrm{kA}$ (see Fig. 8). In order to reduce these energy losses, the additional four 1-MA LTD stages utilized in the present work were built with finer lamination and larger cross section cores. Namely, the cores were fabricated with $50-\mu$-thick tape, and the iron cross section was increased from $\sim 56 \mathrm{~cm}^{2}$ to $\sim 64 \mathrm{~cm}^{2}$. According to expression (4), such changes would increase the resistance $R_{C}$ from $\sim 0.7 \mathrm{Ohm}$ to $\sim 2 \mathrm{Ohm} \mathrm{[24].} \mathrm{The} \mathrm{measured} \mathrm{re-}$ sistance $R_{C}$ of such cores was $\sim 1.5 \mathrm{Ohms}$. Other possible ways to improve the efficiency of the stage is to fabricate the core from Metglas, which is more expensive but has a resistivity $\sim 2.5$ times higher than ET3425 iron and/or use finer lamination core tape.

\section{FIVE-STAGE 1-MA LTD MODULE}

Five 1-MA LTD stages were assembled in series into a module and tested with resistive and electron-beam diode (e-beam diode) loads [25]. In both experiments all the stages were triggered simultaneously, so the LTD module was operated in a LPT mode.

The design of the module with the electron-beam diode load is shown in Fig. 11. The straight cathode stalk has a $757.5-\mathrm{mm}$ radius and is cantilevered. The left side is mounted on the ground flange of the module, while the right freestanding end holds the ring-shaped planar cathode electrode of the diode.

The radial anode-cathode gap around the cathode stalk is $63.5 \mathrm{~mm}$. The peak electric field on the cathode stalk at $\sim 400 \mathrm{kV}$ is $\sim 65 \mathrm{kV} / \mathrm{cm}$, which is well below the vacuum emission threshold of $\sim 200 \mathrm{kV} / \mathrm{cm}$. So the coaxial output vacuum line formed by the five-stage LTD module and the central cathode stalk was designed to operate in the vacuum insulated regime and not in a magnetic insulated transmission line (MITL) regime. The geometric inductance of the coaxial line is $\sim 18 \mathrm{nH}$.

The planar vacuum e-beam diode has a cathode surface area of $\sim 2900 \mathrm{~cm}^{2}$ and an anode-cathode (A-K) gap of 1$2 \mathrm{~cm}$. The A-K is varied by changing the length of the small cylinders which support the annular anode plate. The front surface of the cathode electrode is covered with velvet cloth to facilitate electron emission since the electric field at the cathode was quite low. The diagnostics include two sets of magnetic flux monitors $(d B / d t), \mathrm{B} 1$ and $\mathrm{B} 2$. The two B1 probes (wound in opposite directions relative to each other) are located on the left grounded flange of the module vacuum chamber and when integrated give the total current $I_{C S}$ flowing through the module from the left ground flange to the anode right flange. If there are 


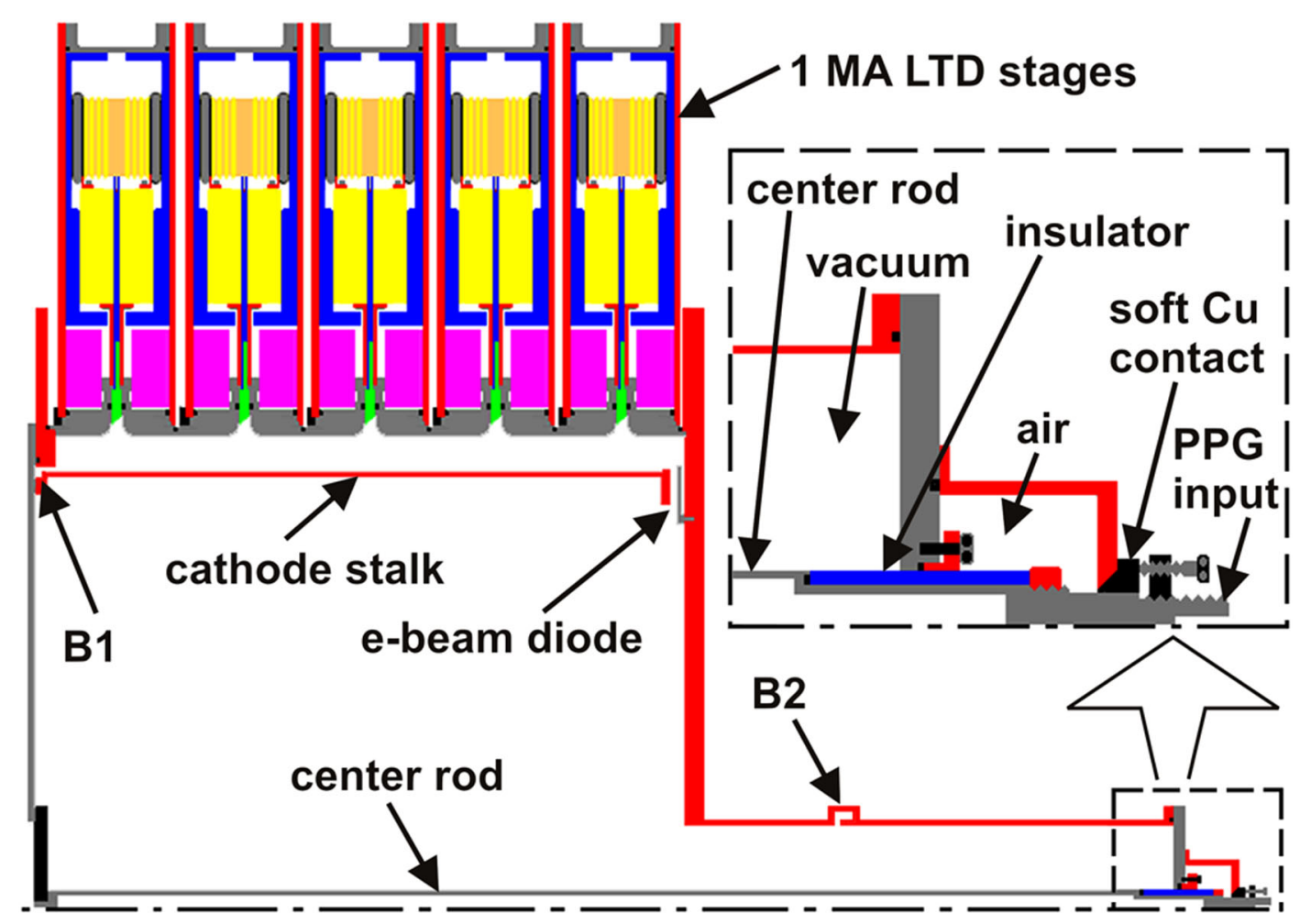

FIG. 11. (Color) Design of the five-stage LTD module with e-beam diode load. The cores of the stages were premagnetized before each shot without opening the module to the atmosphere; the PPG pulse was applied to the right end of the center rod that was disconnected from the grounded body of the module by removing the soft $\mathrm{Cu}$ contact.

no current leaks from the cathode stalk, this current is the sum of the diode current $I_{D}$ and the current flowing through the center rod $I_{C R}$ which shunts the diode with an inductance of $\sim 1085 \mathrm{nH}$. The signals of the two B2 probes (also wound in opposite directions) when integrated provide the current $I_{C R}$ and the diode current $I_{D}=I_{C S}-I_{C R}$ and, when multiplied with the rod inductance, give the diode voltage $U_{D}=L\left(d I_{C R} / d t\right)$.

The center rod serves two purposes: it allows measuring the diode voltage when connected to the right side anode flange, and it also allows premagnetizing the module cores when connected to the premagnetizing power supply (PPG). The design provides the capability to disconnect it between shots from the grounded body of the module for passive premagnetizing of the LTD cores without opening the module chamber to the atmosphere (please see blowup inset in Fig. 11).

The overall design of the five-stage LTD module with the resistive load was the same as in Fig. 11. However, the cathode stalk and the cathode and anode rings were replaced now by a resistive load assembly consisting of 30 separate resistors made from $4.4-\mathrm{cm}$ diameter, $36-\mathrm{cm}$ long polyethylene tubes filled with $\mathrm{KBr}$ water solution. The resistors were mounted at the ends of 3-cm diameter, 67$\mathrm{cm}$ long aluminum rods arranged evenly in a cylindrical array surrounding the axis of the module at a radius of $76.5 \mathrm{~cm}$. Current shunts in series with the resistors were used to measure the current flowing in the $\mathrm{KBr}$ resistors and the voltage across the load. The entire inner volume of the module containing the $\mathrm{KBr}$ resistors assembly was filled with $\mathrm{SF}_{6}$ at atmospheric pressure. Below we present experimental results and numerical simulations for the diode load experiments only.

Figure 12 shows the diode voltage $U_{D}$ (in green) and current $I_{D}$ (in blue) traces for five-stage LTD assembly charged to $U_{C H}= \pm 90 \mathrm{kV}$ and a diode A-K gap of $1.4 \mathrm{~cm}$. In black and red are the diode voltage and current traces simulated with PSPICE for the same charging and diode parameters. The voltage reaches the maximum value of $\sim 400 \mathrm{kV}$ at $\sim 100 \mathrm{~ns}$. At the same time the current peaks at $\sim 800 \mathrm{kA}$, while the electron-beam power becomes $\sim 320 \mathrm{GW}$.

Since all the stages were triggered simultaneously, the module was simulated in PSPICE as a linear pulsed transformer (LPT). The utilized PSPICE equivalent circuit was composed of five RLC circuits connected in series to the diode load through the 18-nH inductance of the transformer output line. The center rod was connected in parallel to the diode, which according to the geometry of Fig. 11 was simulated as two transmission lines connected in series; the first had a $204 \mathrm{Ohm}$ impedance and $3.86 \mathrm{~ns}$ transmission time length and the second had $100 \mathrm{Ohm}$ impedance and $2.96 \mathrm{~ns}$ length, which give a total equivalent inductance of $\sim 1085 \mathrm{nH}$. One of the five RLC circuits was 


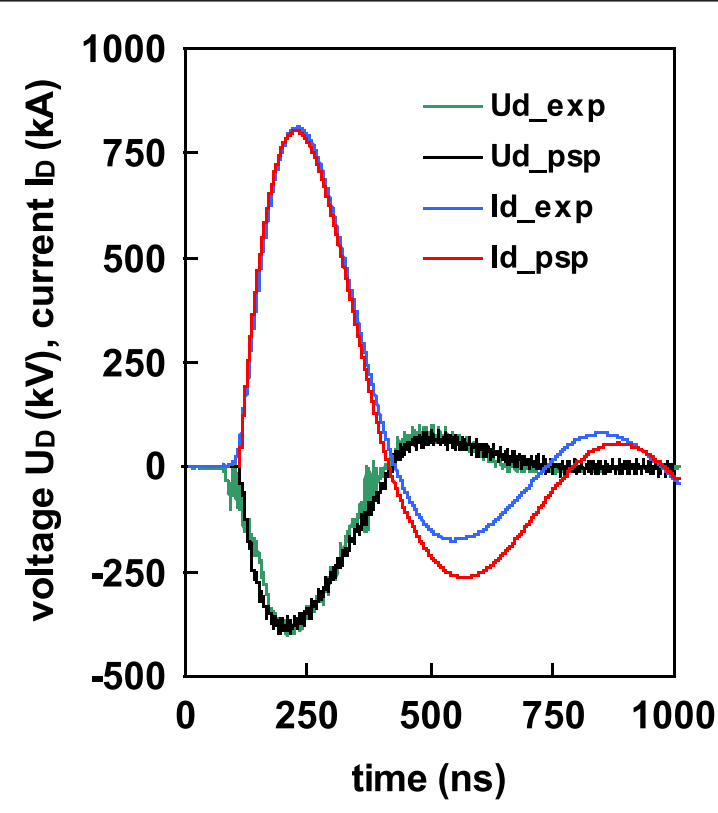

FIG. 12. (Color) Experimental results and PSPICE simulations. The diode voltage $U_{D}$ for clarity of presentation is inverted (negative). The simulations were done for $U_{C H}= \pm 90 \mathrm{kV}$ and an emitting cathode area $A=2100 \mathrm{~cm}^{2}$. The cathode emitting surface was assumed to be moving towards the anode at a constant velocity $V=2 \times 10^{6} \mathrm{~cm} / \mathrm{s}$, simulating the expansion of the cathode plasma.

exactly the same as that presented in Fig. 10 with $R_{C}=$ $0.65 \mathrm{Ohm}$. The other four circuits have a core resistance $R_{C} \sim 2.9$ times larger and equal to $1.9 \mathrm{Ohm}$ because the remaining four stages have been built with finer lamination cores of $50-\mu$-thick iron tape and increased $\sim 64-\mathrm{cm}^{2}$ cross section.

The diode was simulated as a nonrelativistic planar diode with space charge limited electron emission, where the current is defined by

$$
I_{D}=P\left(U_{D}\right)^{3 / 2} \quad \text { where } P=2.335 \times 10^{-6} \frac{A}{d(t)^{2}} .
$$

Here $A$ is the cathode emitting area. The A-K gap $d(t)$ was assumed decreasing with time according to the expression

$$
d(t)=d_{0}-V t
$$

due to cathode plasma expansion at a constant velocity $V=2 \times 10^{6} \mathrm{~cm} / \mathrm{s}$. The initial gap was assumed $1.4 \mathrm{~cm}$. It was allowed to decrease to $0.02 \mathrm{~cm}$ and then was kept constant. To fit the experimental data during the main pulse, the cathode emitting area was assumed to be $A=$ $2100 \mathrm{~cm}^{2}$, which is $\sim 70 \%$ of the geometrical surface area of the cathode.

In the tests with the e-beam diode, the five-stage module was operated at charge voltage between \pm 80 to $\pm 90 \mathrm{kV}$ and a number of AK gap settings varying from 1.0 to $1.7 \mathrm{~cm}$. During the resistive load experiments we fired a total of 500 shots; 300 of them were at $\pm 100 \mathrm{kV}$ charging.

\section{CONCLUSION}

In the present work we have shown that fast LTD stages can be built with as many as 40 separate bricks. The optimum load resistance of this stage is $\sim 0.1 \mathrm{Ohm}$. The power pulse delivered to such a load has a $\sim 100 \mathrm{GW}$ peak value and a rise time of $\sim 100 \mathrm{~ns}$. The electrical behavior of the stage can be predicted by using a simple RLC circuit, which confirms that the jitter of the switches is small compared to the rise time of the output pulse. This greatly simplifies the design of various LTD-based accelerators. The stages can be assembled in series into a module and used as a high-voltage, high-power driver for various applications. The most powerful LTD module consisting of five 1-MA LTD stages was tested with resistive and e-beam diode loads in LPT mode. The experimental results are in excellent agreement with numerical simulations. Experiments with a $1 \mathrm{TW}$ module, which includes 10, 1MA LTD stages connected in series, are in preparation at Sandia National Laboratory, Albuquerque, USA. This LTD module will be the first ever IVA built with a transmission line insulated with deionized water. All ten LTD stages were designed and manufactured at the High Current Electronic Institute (HCEI) in Tomsk, Russia, and transferred to Sandia. Results of this work will be presented in future publications.

\section{ACKNOWLEDGMENTS}

The authors are deeply indebted to their colleagues at the High Current Electronic Institute at Tomsk, Russia, Sandia National Laboratories at Albuquerque, New Mexico, and the International Technologies for High Pulsed Power, Hameau de Drele, Thégra, France. Sandia is a multiprogram laboratory operated by Sandia Corporation, a Lockheed Martin Company, for the U.S. Department of Energy under Contract No. DE-AC04-94AL85000.

\section{APPENDIX A: PREMAGNETIZING OF THE CORE}

When the LTD and/or PPG pulse are applied to the single-turn winding of the core, which is made up by the PPG input and the cavity walls, the magnetic induction $B$ in the core iron varies according to Faraday's law,

$$
B=\frac{1}{S} \int_{0}^{t} U_{\mathrm{LTD}} d t+B_{0}, \quad \text { or } \quad B=\frac{1}{S} \int_{0}^{t} U_{\mathrm{PPG}} d t+B_{0},
$$

where $B_{0}$ is the initial induction at $t=0, S$ is the cross section of iron in the core, $U_{\mathrm{LTD}}$ and $U_{\mathrm{PPG}}$ are the LTD and PPG voltage pulses, respectively. The units of the parameters are in the SI system. If, for example, we assume that the LTD pulse is positive so that it moves the induction along the hysteresis curve in the $+B_{S}$ direction (please see Fig. 13), the PPG pulse (at either passive or active premagnetizing) must be negative to move the induction back 


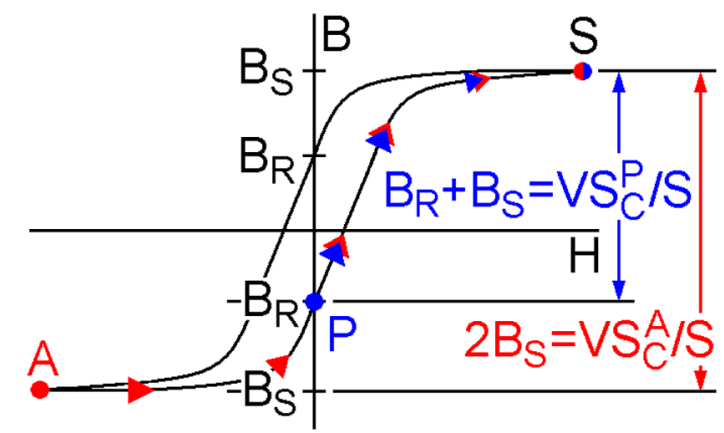

FIG. 13. (Color) Variation of the core induction depending on the LTD firing time relative to the premagnetizing pulse. Blue arrows describe the core behavior if the LTD stage is fired later than the end of the PPG pulse (passive premagnetizing), while the red arrows present the case where the LTD is fired at peak PPG current (active premagnetizing).

down to negative saturation $-B_{S}$. The core saturates when the value of $B$ in Eq. (A1) reaches $B_{S}$ with the LTD pulse and $-B_{S}$ with the PPG pulse, where $B_{S}=2.0 \mathrm{~T}$ is the induction at saturation of ET3425 iron. The difference between the passive and active premagnetizing is the value of the initial induction $B_{0}$ at the time the LTD is fired.

At passive premagnetizing the LTD pulse is applied after the end of the PPG pulse. In this case the induction reaches $-B_{S}$ during the PPG pulse and relaxes to $-B_{R}$ after the end of the pulse. Then for the LTD pulse the initial induction is $B_{0}=-B_{R}$, and the core will not saturate before the voltsecond integral $V S_{\mathrm{LTD}}$ reaches the limit

$$
V S_{\mathrm{LTD}}=\int_{0}^{t} U_{\mathrm{LTD}} d t=S\left(B_{S}+B_{R}\right) \equiv V S_{C}^{P}
$$

The tested 1-MA LTD stage had a core with $S \sim 56 \times$ $10^{-4} \mathrm{~m}^{2}, B_{S}=2 \mathrm{~T}$, and $B_{R}=1.2 \mathrm{~T}$. Equation (A2) then gives $V S_{C}^{P} \sim 18 \mathrm{mVs}$. Passive premagnetizing is the standard method used in modern IVAs.

The induction at saturation $B_{S}(=2.0 \mathrm{~T})$ of the ET3425 iron is sufficiently higher than the residual induction $B_{R}$ $(=1.2 \mathrm{~T})$. This allows an increase of the volt-second integral $V S_{\mathrm{LTD}}$ to a higher than $V S_{C}^{P} \sim 18 \mathrm{mVs}$ value if active premagnetizing is used. At active premagnetizing, the LTD is triggered at the time when the PPG voltage pulse saturates the core in opposite polarity. At that time the PPG current reaches maximum. For the LTD pulse the initial induction then is $B_{0} \sim-B_{S}$, and the core does not saturate before the volt-second integral $V S_{\text {LTD }}$ reaches the maximum value of

$$
V S_{\mathrm{LTD}}=\int_{0}^{t} U_{\mathrm{LTD}} d t=S\left(B_{S}+B_{S}\right) \equiv V S_{C}^{A}
$$

which is $\sim 22.4 \mathrm{mVs}$ for the core of the tested 1-MA LTD stage. Active premagnetizing is used in IVAs utilizing Blumlein as a source driving individual cavities [3].

The variation of the induction in the core iron during the LTD pulse, depending on the premagnetizing method, is schematically illustrated in Fig. 13. The blue arrows indicate the path from point $P$ to point $S$ followed by the magnetic induction of the core during the LTD pulse when the LTD is triggered after the PPG pulse (passive premagnetizing). The red arrows indicate the path from point $A$ to point $S$ taken by the core during the LTD pulse when the LTD is triggered at peak PPG current. At that time the induction of the core is at $B_{0} \sim-B_{S}$ (active premagnetizing).

\section{APPENDIX B: OPTIMUM LOAD IMPEDANCE OF AN LTD STAGE}

\section{Derivation of Eq. (5)}

We estimate here the optimum load impedance $R_{\text {opt }}$ of the LTD circuit presented in Fig. 10. Reference [15] finds that when $R_{C} \rightarrow \infty$ and $L_{2} / L_{1}=0$, the optimum load impedance is given by the following expression:

$$
R^{*}=1.1 \sqrt{\frac{L_{1}+L_{2}}{C}}+0.8 R_{1} .
$$

Reference [26] finds that when $R_{C}$ is finite and $L_{2} / L_{1}=0$, the optimum load impedance is given by the following:

$$
R_{\text {opt }}^{\prime}=R^{*}\left[1-0.73 \frac{R^{*}}{R_{C}}\right] .
$$

We estimate here the optimum load impedance $R_{\mathrm{opt}}$ when $R_{C}$ is finite and $L_{2} / L_{1} \neq 0$. We use dimensional analysis to observe that it may be possible to express the ratio $R_{\text {opt }} / R_{\text {opt }}^{\prime}$ as a function only of the ratios $R_{\text {opt }}^{\prime} / R_{C}$ and $L_{2} / L_{1}$ :

$$
\frac{R_{\mathrm{opt}}}{R_{\mathrm{opt}}^{\prime}}=f\left(\frac{R_{\mathrm{opt}}^{\prime}}{R_{C}}, \frac{L_{2}}{L_{1}}\right) .
$$

We calculated $R_{\text {opt }} / R_{\text {opt }}^{\prime}$ numerically at several values of $R^{\prime}{ }_{\text {opt }} / R_{C}$ and $L_{2} / L_{1}$. We find that to a reasonable approximation

$$
R_{\mathrm{opt}}=R^{*}\left[1-0.73\left(1-0.66 \frac{L_{2}}{L_{1}}\right) \frac{R^{*}}{R_{C}}\right] .
$$

When $L_{2} / L_{1}=0$, Eq. (B4) becomes identical to Eq. (B2), which is developed in Ref. [26]. When $L_{2} / L_{1}=0$ and $R_{\text {opt }}^{\prime} / R_{c}=0$, Eq. (B4) becomes identical to Eq. (B1), which is developed in Ref. [15]. Equation (B4) is correct to $\sim 1 \%$ whenever

$$
\begin{gathered}
\frac{R_{1}}{\sqrt{\left(L_{1}+L_{2}\right) / C}}<0.5, \\
\frac{R^{*}}{R_{C}} \leq 0.2, \\
\frac{L_{2}}{L_{1}} \leq 0.3 .
\end{gathered}
$$


[1] N. C. Christofilos, R.E. Hester, W. A.S. Lamb, D. D. Reagan, and W. A. Sherwood, Rev. Sci. Instrum. 35, 886 (1964).

[2] A. I. Anatsky, O. O. Bogdanov, P. V. Bukaev, and Ju. P. Vahrushin, Atomnaya Energia 21, 439 (1966).

[3] I. D. Smith, Phys. Rev. ST Accel. Beams 7, 064801 (2004).

[4] M. G. Mazarakis, J. W. Poukey, S. L. Shope, C. A. Frost, B. N. Turman, J. J. Ramirez, K. R. Prestwich, and P. J. Pankuch, in Proceedings of the 8th IEEE International Pulsed Power Conference, edited by R. White and K. Prestwich (IEEE, Piscataway, NJ, 1991), p. 86.

[5] M. G. Mazarakis, J. W. Poukey, D. Rovang, S. Cordova, P. Pankuch, R. Wavrik, D. L. Smith, J.E. Maenchen, L. Bennett, K. Shimp, and K. Law, in Proceedings of Space Charge Dominated Beams and Applications of High Brightness Beams, edited by S. Y. Lee (Indiana University, Bloomington, IN, 1995), p. 479.

[6] M. G. Mazarakis, J. W. Poukey, D. C. Rovang, J.E. Maenchen, S. R. Cordova, P. R. Menge, R. Pepping, L. Bennett, K. Mikkelson, D. L. Smith, J. Halbleib, W. A. Stygar, and D. R. Welch, Appl. Phys. Lett. 70, 832 (1997).

[7] G. A. Mesyats, in Proceedings of the 2nd IEEE International Pulsed Power Conference, edited by A. H. Guenther and M. Kristiansen (IEEE, Piscataway, NJ, 1979), p. 9.

[8] B. M. Kovalchuk, V. A. Vizir, A. A. Kim, E. V. Kumpyak, S. V. Loginov, A. N. Bastrikov, V. V. Chervjakov, N. V. Tsou, Ph. Monjaux, and D. Huet, Russ. Phys. J. 40, 1142 (1997).

[9] A. A. Kim (unpublished).

[10] M. G. Mazarakis and R. B. Spielman, in Proceedings of the 12th IEEE International Pulsed Power Conference, edited by C. Stallings and H. Kirbie (IEEE, Piscataway, NJ, 1999), p. 412.

[11] A. A. Kim and B. M. Kovalchuk, in Proceedings of the 12th Symposium on High Current Electronics, edited by G. Mesyats, B. Kovalchuk, and G. Remnev (Tomsk Polytechnic University and Institute of High Current Electronics SD RAS, Tomsk, Russia, 2000), p. 263.

[12] M. G. Mazarakis and R. B. Spielman, in Proceedings of the 20th International Linac Conference (Stanford Linear Accelerator Center, Stanford, CA, 2000), p. 497 (Stanford Linear Accelerator Center Report No. SLAC-R-561, edited by A. W. Chao).

[13] M. G. Mazarakis, R. B. Spielman, K. W. Struve, and F. W. Long, in Proceedings of the 13th IEEE International Pulsed Power Conference, edited by R. Reinovsky and M. Newton (IEEE, Piscataway, NJ, 2001), p. 587.

[14] M. G. Mazarakis and K. W. Struve, Sandia National Laboratories Report No. SAND2006-5811, 2006.
[15] W. A. Stygar, M. E. Cuneo, D. I. Headley, H. C. Ives, R. J. Leeper, M. G. Mazarakis, C. L. Olson, J. L. Porter, T. C. Wagoner, and J.R. Woodworth, Phys. Rev. ST Accel. Beams 10, 030401 (2007).

[16] Capacitor Model GA 35426, designed and manufactured especially for the fast LTD by General Atomics Electronic Systems, 4949 Greencraig Lane, San Diego, CA 92123, www.gaep.com.

[17] These switches were designed and manufactured by the Institute of High-Current Electronics in Tomsk, Russia, especially for the fast LTD stages.

[18] A. A. Kim, V. G. Durakov, S. N. Volkov, A. N. Bastrikov, B. M. Kovalchuk, V. A. Sinebryukhov, S. V. Frolov, V. M. Alexeenko, L. Veron, M. Toury, C. Vermare, R. Nicolas, F. Bayol, and C. Drouilly, in Proceedings of the 14th Symposium on High Current Electronics, edited by B. Kovalchuk and G. Remnev (Institute of High Current Electronics SD RAS, Tomsk, Russia, 2006), p. 297.

[19] K. Hahn, J. R. Woodworth, W. T. Clark, J. R. Blickem, R. Starbird, M. J. Hardin, and Y. Maron, in Proceedings of the 16th IEEE International Pulsed Power Conference, edited by E. Schamiloglu and F. Peterkin (IEEE, Piscataway, NJ, 2007), p. 125.

[20] V. A. Sinebryukhov, A. A. Kim, B. M. Kovalchuk, S. V. Frolov, V. M. Alexeenko, and S. N. Volkov, in Proceedings of the 12th International Conference on Megagauss Magnetic Field and Related Topics, Novosibirsk, 2008 (to be published).

[21] MicroSim Schematics, Version 6.1b-November 1994. MicroSim Corporation, 20 Fairbanks, Irvine, California 92718, USA.

[22] V. A. Vizir, A. D. Maksimenko, V. I. Manylov, and G. V. Smorudov, in Proceedings of the 13th Symposium on High Current Electronics, edited by B. Kovalchuk and G. Remnev (Institute of High Current Electronics SD RAS, Tomsk, Russia, 2004), p. 198.

[23] S. S. Vdovin, Design of the Pulsed Transformers (Energia Publishing House, Leningrad, 1971), p. 66 (in Russian).

[24] F. Bayol (private communication).

[25] A. Kim, V. Sinebryukhov, B. Kovalchuk, A. Bastrikov, V. Durakov, S. Volkov, S. Frolov, V. Alexeenko, M. Mazarakis, D. McDaniel, C. Olson, K. Struve, and R. Gilgenbach, in Proceedings of the 16th IEEE International Pulsed Power Conference, edited by E. Schamiloglu and F. Peterkin (IEEE, Piscataway, NJ, 2007), p. 144.

[26] W. A. Stygar, W. E. Fowler, K. R. LeChien, F. W. Long, M. G. Mazarakis, G. R. McKee, J.L. McKenney, J. L. Porter, M.E. Savage, B.S. Stoltzfus, D. M. Van De Valde, and J.R. Woodworth, Phys. Rev. ST Accel. Beams 12, 030402 (2009). 